\title{
The Sinc Function Representation and Three-Loop Master Diagrams
}

\author{
Richard Easther, Gerald Guralnik, and Stephen Hahn \\ Department of Physics, Brown University, Providence, RI 02912, USA.
}

\begin{abstract}
We test the Sinc function representation, a novel method for numerically evaluating Feynman diagrams, by using it to evaluate the three-loop master diagrams. Analytical results have been obtained for all these diagrams, and we find excellent agreement between our calculations and the exact values. The Sinc function representation converges rapidly, and it is straightforward to obtain accuracies of 1 part in $10^{6}$ for these diagrams and with longer runs we found results better than 1 part in $10^{12}$. Finally, this paper extends the Sinc function representation to diagrams containing massless propagators.
\end{abstract}

BROWN-HET-1192

\section{Introduction}

The Sinc function representation, introduced by us in Ref. [1] , is a new approach to numerically evaluating Feynman diagrams. This paper tests the Sinc function representation by using it to calculate the three-loop master diagrams, whose analytical forms were obtained by Broadhurst in a mathematical tour de force [2]. We have selected these diagrams because, in order to make a meaningful test of the Sinc function representation, we must apply it to diagrams which contain multiple loops and which have been evaluated analytically, so that we can compare our results to known exact values. The criteria "topologically complex" and "evaluated analytically" are not mutually compatible, but the three-loop master diagrams are sufficiently complex to allow a stringent test of our methods. In addition, the master diagrams contain massless lines, so we must start by extending the Sinc function representation to theories with massless propagators.

The Sinc function representation performs an ab initio computation of the corresponding integral, so we make no use of the special analytic properties of the master diagrams. Consequently, our success with these diagrams supports our more general claim that the Sinc function representation is a powerful tool for accurately evaluating arbitrary complex diagrams.

We believe that our results, which are contain up to 12 or 13 significant figures, represent an unprecedented level of precision for the numerical evaluation of a non-trivial Feynman integral. Conventional wisdom holds that multidimensional Feynman integrals must be numerically evaluated with Monte Carlo methods, of which VEGAS is the canonical implementation [3]. Monte Carlo methods usu- ally yield the first few significant figures without too much difficulty but their efficiency drops extremely rapidly as the desired accuracy is increased, making highly accurate calculations effectively impossible.

Obviously, in any realistic situation these diagrams correspond to higher order corrections, and we are capable of a level of precision far beyond than that of any experimental data. However, if only the first few significant figures of the exact result are need the Sinc function representation can compute these very quickly. Furthermore, Broadhurst's analytical treatment of the master diagrams relies on the PSLQ algorithm [4] for identifying integer relationships. Part of input required by this algorithm requires is accurate numerical evaluation of the diagrams. By first reducing the master diagrams to one- (or two-) dimensional integrals, Broadhurst was able to extract the numerical results he needed. However, if one wished to apply the same techniques to diagrams that cannot be reduced to numerical integrals which are tractable using standard methods, the Sinc function representation provides a promising alternative approach for their numerical evaluation.

In the following section we summarize the Sinc function representation, and show how it can be applied to diagrams with both massive and massless propagators. In Section 3 we define the three-loop master diagrams and derive their Sinc function representations. We evaluate these diagrams in Section 4 and compare our results with the analytic forms, while in Section 5 we discuss the implications of our work. 


\section{Sinc Function Representation}

The Sinc function representation [1] hinges upon a novel representation of the propagator and, as the name suggests, is derived using the properties of the generalized Sinc function,

$$
S_{k}(h, x)=\frac{\sin [\pi(x-k h) / h]}{\pi(x-k h) / h},
$$

where the capital $S$ distinguishes this from the more familiar form, $\operatorname{sinc}(x)=\sin (x) / x$. The properties of the $S_{k}(h, x)$ are thoroughly discussed by Stenger [5]. For our purposes, their most important attributes are that any function $f(z)$ which is analytic on an infinite strip of the complex plane that includes the real axis can be approximated as

$$
f(z) \approx \sum_{k=-\infty}^{\infty} f(k h) S_{k}(h, z),
$$

while

$$
\int_{-\infty}^{\infty} f(z) d z \approx \sum_{k=-\infty}^{\infty} f(k h) .
$$

Crucially, the accuracy of the approximation improves exponentially as $h \rightarrow 0$ [5]. In Ref. [1] we constructed the Sinc function representation for the scalar field propagator which, in Euclidean momentum space, has the form

$$
G_{\Lambda}(p)=\frac{1}{p^{2}+m^{2}} e^{-p^{2} / \Lambda^{2}}
$$

where we have introduced a cutoff in anticipation of divergent integrals. We now derive a version of the Sinc function representation which is applicable to both massive and massless fields.

The spacetime propagator is the Fourier transform of $G_{\Lambda}(p)$

$$
\begin{array}{r}
G_{\Lambda}(x)=\frac{1}{(4 \pi)^{2}} \int_{0}^{\infty} d s \frac{1}{\left(s^{2}+\frac{1}{\Lambda^{2}}\right)^{2}} \times \\
\exp \left(-m^{2} s-\frac{x^{2}}{s^{2}+\frac{1}{\Lambda^{2}}}\right),
\end{array}
$$

where we introduced $s$ by exponentiating the denominator, and then performed the Fourier transform. We now replace $s$ by $e^{z}$ and use equation (3) to approximate the integral over $z$, which yields the Sinc function representation of the propagator,

$$
\begin{gathered}
G_{\Lambda h}(x)=\frac{h}{(4 \pi)^{2}} \sum_{k=-\infty}^{\infty} \frac{\exp \left(k h-m^{2} e^{k h}\right)}{\left(e^{k h}+\frac{1}{\Lambda^{2}}\right)^{2}} \times \\
\exp \left(-\frac{x^{2}}{e^{k h}+\frac{1}{\Lambda^{2}}}\right) .
\end{gathered}
$$

The massless limit of equation (6) is obvious, and setting $m=0$ makes no difference to its impressive convergence properties [1]. The difference between this form of $G_{\Lambda h}$ and the form found in Ref. [1] is largely aesthetic: when $m \neq 0$, rescaling $s$ to $s / m$ makes all the terms inside the exponentials explicitly dimensionless. Lastly, it will be convenient to write $G_{\Lambda h}$ as

$$
G_{\Lambda h}(a-b)=\frac{h}{(4 \pi)^{2}} \sum_{k=-\infty}^{\infty} p(k) \exp \left[-\frac{(a-b)^{2}}{4 c(k)}\right]
$$

where

$$
\begin{aligned}
& c(k)=e^{k h}+\frac{1}{\Lambda^{2}} \\
& p(k)=\frac{\exp \left(k h-m^{2} e^{k h}\right)}{\left(e^{k h}+\frac{1}{\Lambda^{2}}\right)^{2}}=\frac{\exp \left(k h-m^{2} e^{k h}\right)}{c(k)^{2}} .
\end{aligned}
$$

Feynman diagrams are integrals over products of propagators, and we derive the Sinc function representation for a given diagram by inserting $G_{\Lambda h}$ for each line. All the spacetime integrals required by the internal vertices can be performed analytically, and we are left with an $N$-dimensional infinite sum which approximates the original integral, where $N$ is the number of internal lines in the diagram. In Ref. [1] we give Sinc function Feynman rules which describe the construction the Sinc function representation for an arbitrary diagram, and these are not changed by the inclusion of the massless propagators.

\section{The Master Diagrams}

The 10 three-loop master diagrams, or vacuum tadpoles, are depicted in Figure 11. In addition to their intrinsic beauty, their practical use is that three-loop diagrams dominated by a single, large mass and with small external momenta can be expressed in terms of the master diagrams. Four of these diagrams were given analytically before 1998 [6 9], and Broadhurst found results for the remaining six [2]. Applying the coordinate space Feynman rules to the overall "tetrahedron" topology of the master diagrams yield the following integral

$$
\begin{gathered}
V_{j}(\Lambda)=(4 \pi)^{6} \int d^{4} x_{1} d^{4} x_{2} d^{4} x_{3} G_{1}\left(x_{1}-x_{2}\right) \\
G_{2}\left(x_{1}-x_{3}\right) G_{3}\left(x_{1}-x_{4}\right) G_{4}\left(x_{2}-x_{3}\right) \\
G_{5}\left(x_{2}-x_{4}\right) G_{6}\left(x_{3}-x_{4}\right) .
\end{gathered}
$$

The subscript $j$ labels the specific combination of massive and massless propagators, as enumerated in Figure 1, 

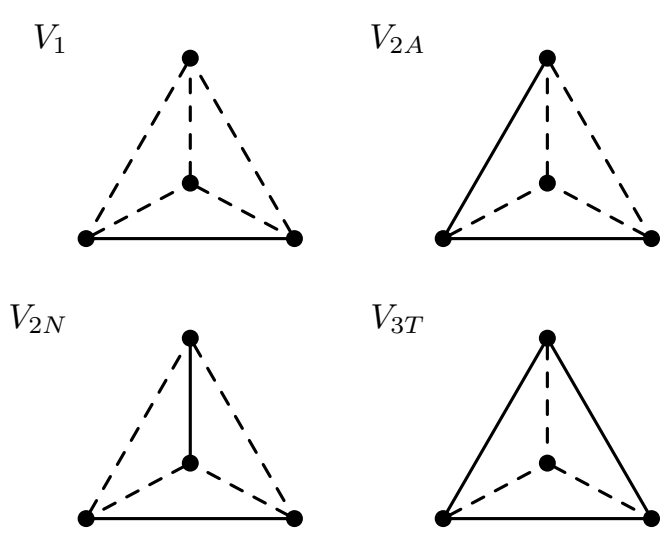

$V_{3 T}$

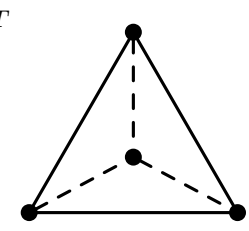

$V_{3 S}$

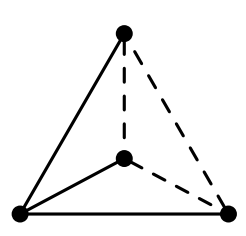

$V_{4 A}$

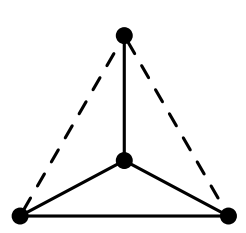

$V_{5 N}$

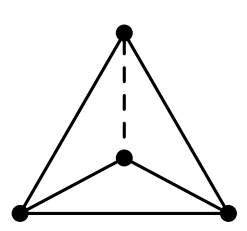

$V_{3 L}$

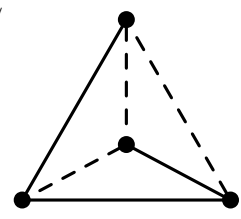

$V_{4 N}$

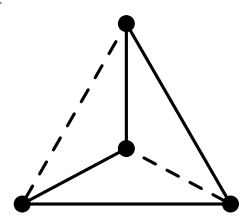

$V_{6}$

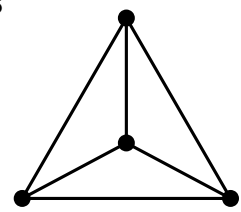

Figure 1: The three-loop master diagrams, where dashed lines are massless.

while the prefactor reconciles our measure with Broadhurst's. Equation (10) is a coordinate space integral, but this analysis works equally well in momentum space. The vertex labels are shown in Figure 2, and from here on we use Lorentz invariance to set $x_{4}$ to zero. The six propagators are functions of the mass, $m_{j}$, carried by each line. For the master diagrams, the $m_{j}$ are either 0 or 1 , but the Sinc function representation yields results even if all the non-zero masses are different. The integral $V_{j}$ is divergent, but it is regulated by employing the cut-off propagator, $G_{\Lambda}(x)$.

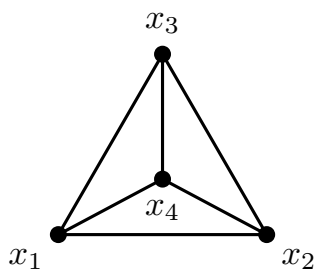

Figure 2: The generic topology of the master diagram, showing our choice of vertex labels.

Applying the Sinc function Feynman rules, we insert $G_{\Lambda h}$ for each of the propagators, and perform the Gaussian integrals. After some algebraic manipulation, we obtain the the Sinc function representation of the general integral $V_{j}$,

$$
V_{j}(\Lambda, h)=h^{6} \sum_{k} \frac{\prod_{n=1}^{6} c_{n}\left(k_{n}\right)^{2} p_{n}\left(k_{n}\right)}{\left(a+b c_{6}\left(k_{6}\right)\right)^{2}},
$$

where

$$
\begin{aligned}
a= & \left(c_{2}\left(k_{2}\right)+c_{5}\left(k_{5}\right)\right) c_{3}\left(k_{3}\right) c_{4}\left(k_{4}\right)+ \\
& c_{2}\left(k_{2}\right) c_{5}\left(k_{5}\right)\left(c_{3}\left(k_{3}\right)+c_{4}\left(k_{4}\right)\right)+ \\
& c_{1}\left(k_{1}\right)\left(c_{2}\left(k_{2}\right)+c_{3}\left(k_{3}\right)\right)\left(c_{4}\left(k_{4}\right),+c_{5}\left(k_{5}\right)\right) \\
b= & \left(c_{2}\left(k_{2}\right)+c_{4}\left(k_{4}\right)\right)\left(c_{3}\left(k_{3}\right)+c_{5}\left(k_{5}\right)\right)+ \\
& c_{1}\left(k_{1}\right)\left(c_{2}\left(k_{2}\right)+c_{3}\left(k_{3}\right)+c_{4}\left(k_{4}\right)+c_{5}\left(k_{5}\right)\right) .
\end{aligned}
$$

The $c_{i}$ and $p_{i}$ are defined by equations (8) and (9) and the summation over $k$ is shorthand for infinite sums running between $\pm \infty$ for each $k_{i}, i=1 \cdots 6$.

To compute $V_{j}$ from the Sinc function representation we numerically evaluate the six-dimensional infinite sum in equation (11). In practice, $V_{j}(\Lambda, h)$ is an approximation to $V_{j}(\Lambda)$, which is exact in the limit $h \rightarrow 0$ [1]. By choosing $h$, we effectively fix the accuracy with which it is possible to evaluate $V_{j}$. Moreover, although the cutoff renders $V_{j}$ finite, the result of evaluating equation (11) directly depends on $\Lambda$, and we must remove this regularization dependence before proceeding.

Examining the six-dimensional sum, we see that if $\Lambda \rightarrow 0$ and $k_{i}=k$, then the general term approaches a constant, $h^{2} /\left(256(4 \pi)^{6}\right)$, as $k$ becomes large and negative. Consequently, the sum diverges in the limit $\Lambda \rightarrow \infty$, and we have thus identified the origin of the divergent part of $V_{j}$ in the Sinc function representation.

In Ref. 11, we renormalized the Sinc function representation of the sunset graph by expanding it about its external momentum and subtracting the $p^{0}$ and $p^{2}$ terms. 


\begin{tabular}{|r||l|l|l|}
\hline$j$ & $F_{j}$ (exact) & $F_{j}$ (computed) & Error \\
\hline \hline $2 A$ & 8.65858586969 & 8.65857982635 & $-6.9810^{-7}$ \\
$2 N$ & 9.33906272305 & 9.33905792236 & $-5.1410^{-7}$ \\
$3 T$ & 12.9878788045 & 12.98787117 & $-5.8810^{-7}$ \\
$3 S$ & 13.3201733442 & 13.3201656342 & $-5.7910^{-7}$ \\
$3 L$ & 13.4863206141 & 13.4863128662 & $-5.7410^{-7}$ \\
$4 A$ & 16.372515904 & 16.3725070953 & $-5.3810^{-7}$ \\
$4 N$ & 16.5134789787 & 16.513469696 & $-5.6210^{-7}$ \\
$5 N$ & 18.6761709376 & 18.6761627197 & $-4.410^{-7}$ \\
6 & 20.4945895999 & 20.4945793152 & $-5.0210^{-7}$ \\
\hline
\end{tabular}

Table 1: We have computed the 9 non-trivial $F_{j}$ and compared them to the exact values. These results were obtained with $h=0.7$.

This approach cannot be used with the master diagrams as they do not carry external momenta. However, the divergent part does not depend on any of the masses in the diagram. Consequently, the difference of any two $V_{j}(\Lambda, h)$ is finite even when $\Lambda \rightarrow \infty$, as the divergent parts in the sums for each of the $V_{j}(\Lambda, h)$ will cancel term by term. In an approach that parallels Broadhurst's analytical treatment of the $V_{j}$, we write the differences between $V_{j}$ and $V_{0}$ as

$$
F_{j}(h)=\sum_{k_{i}} \lim _{\Lambda \rightarrow \infty}\left(t_{1}-t_{j}\right)
$$

where $t_{j}$ is the general term from the six-dimensional sum that gives the Sinc function representation of $V_{j}$. By forming the difference between $V_{1}$ and $V_{j}$ in this way, and then taking the limit $\Lambda \rightarrow \infty$, we compute the finite $F_{j}$ without ever needing to evaluate the $V_{j}(\Lambda)$, which diverge in the limit $\Lambda \rightarrow \infty$. By taking this approach we avoid the loss of precision that would result from attempting to evaluate $F_{j}$ by computing $V_{j}$ at large but finite $\Lambda$ and then subtracting the result from $V_{1}$.

In Figure 11 we have used the symmetry of the diagrams to ensure that $G_{1}$, the propagator between $x_{1}$ and $x_{2}$, is always massive. Since all the other lines in $V_{1}$ are massless and $p_{k}$ with $m^{2}>0$ is always less than $p_{k}$ with $m^{2}=0$, our choice of labeling ensures that $t_{1}>t_{j}$ for any $\left\{k_{1}, \cdots, k_{6}\right\}$. Thus all the terms in $F_{j}$ are positive, and a numerical evaluation of the multidimensional sum approaches the limiting value monotonically.

We could be content with simply calculating the differences between diagrams and comparing our result to the analytic values. However, we can make use of a single analytical result, namely that the finite part of $V_{1}$ is $6 \zeta(3)+3 \zeta(4)$, to relate our Sinc function representation calculation of the $F_{j}$ with the renormalized $V_{j}$.

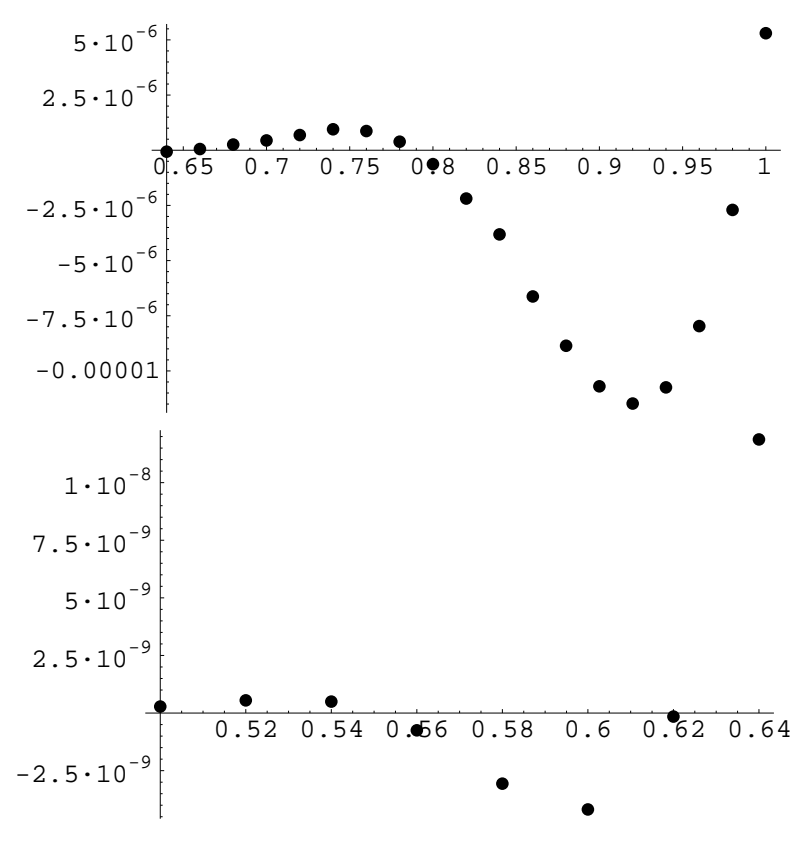

Figure 3: We plot $1-F_{10}(h) / F_{10}$, as a function of $h$.

\section{Numerical Evaluations}

We evaluate the $F_{j}$ with a code that is similar to those used to obtain the results given in Ref. [1]. T) The code adaptively limits itself to the region of the six-dimensional lattice defined by the $k_{i}$ that make the strongest contribution to the overall sum. We accelerate the convergence of each of the six sums by using an Aitken $\delta^{2}$ extrapolation [1, 10]. This can significantly reduce the time needed to evaluate the sums, but the problem is still tractable without the extrapolation. While we have endeavored to ensure that the algorithm is implemented efficiently, there is no guarantee that it is the optimal method for evaluating the Sinc function representation of $F_{j}$. Consequently, the timing data we present (obtained using a $250 \mathrm{MHz}$ UltrasPARC II CPU) should be interpreted as a upper limit to the effort needed to evaluate these diagrams' Sinc function representations.

When using equation (14) to evaluate the $F_{j}$, we find that there are two distinct sources of error. The first is the intrinsic difference between $F_{j}(h)$ and the exact value of $F_{j}$, and the second is the numerical error (if any) in

${ }^{1}$ Sample codes for calculating $F_{\text {; }}$ can be found at the URL http://www.het.brown.edu/people/easther/feynman// 
the evaluation of the infinite series. This has two possible origins: either because the summation was truncated prematurely, or because of an accumulation of numerical error. In Table 1, we show the results for the nine nontrivial $F_{j}$, with $h=0.7$. It is comparatively easy for us to obtain results accurate to within a few parts in $10^{7}$.

The difference between the Sinc function representation and the exact value decreases quickly as $h$ is reduced. In Figure 3, we plot the error in the value of $F_{10}$ computed from the Sinc function representation, as a function of $h$. The effort required to evaluate the sum increases at smaller $h$. First, the general term dies away more slowly when $h$ is decreased, so we must add more terms to evaluate the Sinc function representation to a given level of accuracy. In addition, with smaller values of $h$, the maximum size of the terms which can be ignored by the numerical algorithm also drops, which further increases the effort needed to compute the multi-dimensional sums. With $h \sim 1.0$, the Sinc function representation could be evaluated in approximately three minutes on a workstation. The exact timings depend on the truncation procedure used in our code, which is somewhat heuristic and probably too conservative. With $h \sim 0.7$, the evaluation time rises to around 20 minutes, and with $h \sim 0.5$, several hours are required.

By paying close attention to the form of the sum, it is possible to improve on these speeds. We are particularly interested in computing $F_{6}$, the master diagram in which all lines are massive. A direct analytical treatment of this diagram yields a double integral, which must then be computed numerically in order to verify the results obtained with the PSLQ algorithm [2]. All the other results identified this way can be compared to (at worst) one dimensional integrals, which could be computed to very high precision. However, the result for $F_{6}$ has not been checked beyond 15 decimal places.

We can make two improvements to the efficiency of the Sinc function representation of $F_{6}$. First, we can compute $V_{5 N}-V_{6}$, instead of $F_{6}$, as the sums in the positive $k_{i}$ directions for $i=2, \cdots, 5$ converge much more rapidly than those in $F_{6}$, due to the $\exp \left(-e^{k_{i} h}\right)$ terms contributed by the extra massive propagators in $V_{5 N}$. Since $V_{5 N}$ is known analytically, we can then deduce both $V_{6}$ and $F_{6}$. Second, we can speed the evaluation of the inner loop since we can write equation (11) as

$$
V_{j}(\Lambda, h)=h^{6} \sum_{k_{1} \cdots k_{5}}\left[\frac{\prod_{n=1}^{5} c_{n}\left(k_{n}\right)^{2} p_{n}\left(k_{n}\right)}{b^{2}} f\left(\frac{a}{b}\right)\right]
$$

where

$$
f\left(\frac{a}{b}\right)=\sum_{k_{6}} \frac{c_{6}\left(k_{6}\right)^{2} p_{6}\left(k_{6}\right)}{\left(a / b+c_{6}\left(k_{6}\right)\right)^{2}},
$$

and we recall that $b$ does not depend on $k_{6}$. Since $f(a / b)$ depends on the single parameter $a / b$ we can construct an interpolation table which allows us to evaluate $f$ in far less time than it takes to compute the sum over $k_{6}$. This effectively reduces the problem to a five-dimensional sum. The combination of these two methods improves the evaluation time by an order of magnitude or more, and it is straightforward to calculate $V_{6}$ to within a few parts in $10^{13}$.

Unfortunately, going beyond 16 digits of precision requires the use of quadruple precision variables, and this dramatically lowers the computational efficiency of the code on present hardware. However, given a machine that performed 128-bit numerical arithmetic at similar speeds to 64 -bit arithmetic, we would be able to evaluate $F_{6}$ to a precision much better than 1 part in $10^{-16}$.

\section{Discussion}

The immediate purpose of this paper was to show that the Sinc function representation reproduces the analytic results for the three-loop master diagrams. Since our results are limited only by the finite accuracy of double-precision computer arithmetic, we have emphatically demonstrated the efficacy of the Sinc function representation in this specific case. We pursued this problem to test the usefulness of the Sinc function representation as a tool for evaluating more general Feynman integrals. The Sinc function representation does not rely on any special properties of the diagrams being evaluated, and we made no use of the analytic knowledge gained from previous work on the master diagrams. Thus, these results support our claim that the Sinc function representation may be a useful approach to evaluating general higher order diagrams [1].

There is considerable theoretical interest in performing accurate calculations of specific diagrams to assist the investigation of non-trivial relationships between diagrams. Broadhurst's evaluation of the three-loop master diagrams is a prime example of this type of work, which has the ability to illuminate the deep structure of perturbative quantum field theory. The Sinc function representation has the potential to facilitate this approach, since it is fast, accurate, and does not require a partial analytical evaluation of the diagram.

When analyzing experimental data, we are unlikely to need the high levels of accuracy achieved in this paper. 
However, if we only want a few significant figures, we can increase $h$, which allows us to evaluate diagrams very rapidly. The Sinc function representation's convergence properties, lack of analytic overhead, and applicability to arbitrary topologies suggests that it may be a useful tool for automatically calculating large sets of diagrams. However, before this possibility can be explored in detail, we must generalize the Sinc function representation to fermionic and vector fields, and describe a renormalization procedure that can be applied to the Sinc function representation of an arbitrary diagram. This work is currently in progress.

Finally, while Feynman integrals arise in perturbative quantum field theory, the Sinc function representation is an outgrowth of a new approach to "exact" numerical quantum field theory, the Source Galerkin method 111 16. To apply the source Galerkin method we must carry out integrals over products of terms proportional to the free propagator, which resemble Feynman integrals. Consequently, the numerical codes and analytical insight needed to apply the Sinc function representation to Feynman integrals also improve the efficiency of the Source Galerkin method.

\section{Acknowledgments}

We thank Gyan Bhanot for a useful discussion. Computational work in support of this research was performed at the Theoretical Physics Computing Facility at Brown University. This work is supported by DOE contract DEFG0291ER40688, Tasks A and D.

\section{References}

[1] R. Easther, G. Guralnik, and S. Hahn, hep-ph/9903255 (1999).

[2] D. Broadhurst, Eur. Phys. J. C8, 311 (1999).

[3] G. P. Lepage, J. Comput. Phys. 27, 192 (1978).

[4] H. R. P. Ferguson, D. H. Bailey, and S. Arno, Math. Comput. 68, 351 (1999).

[5] F. Stenger, Numerical Methods Based on Sinc and Analytic Functions (Springer Verlag, New York, NY, 1993).

[6] K. G. Chetyrkin, A. L. Kataev, and F. V. Tkachov, Phys. Lett. B 99, 147 (1981).

[7] F. V. Tkachov, Phys. Lett. B 100, 65 (1981).
[8] D. Broadhurst, Z. Phys. C 54, 599 (1992).

[9] L. V. Avdeev, Comput. Phys. Commun. 98, 15 (1996).

[10] W. Press, S. Teukolsky, W. Vetterling, and B. Flannery, Numerical Recipes in Fortran, 2 ed. (Cambridge UP, Cambridge, 1992).

[11] S. García, G. S. Guralnik, and J. W. Lawson, Phys. Lett. B 333, 119 (1994).

[12] S. García, Z. Guralnik, and G. S. Guralnik, hepth/9612079 (1996).

[13] J. W. Lawson and G. S. Guralnik, Nucl. Phys. B 459, 589 (1996).

[14] S. C. Hahn, Ph.D. thesis, Brown University, 1998.

[15] S. C. Hahn and G. S. Guralnik, hep-th/9901019 (1999).

[16] P. Emirda ̆̆, R. Easther, G. Guralnik, and S. Hahn, hep-lat/9909122 (1999). 